\title{
Accelerating numerical solution of Stochastic Differential Equations with CUDA
}

\author{
M. Januszewski, M. Kostur ${ }^{\mathrm{a}}$ \\ ${ }^{a}$ Institute of Physics, University of Silesia, 40-007 Katowice, Poland
}

\begin{abstract}
Numerical integration of stochastic differential equations is commonly used in many branches of science. In this paper we present how to accelerate this kind of numerical calculations with popular NVIDIA Graphics Processing Units using the CUDA programming environment. We address general aspects of numerical programming on stream processors and illustrate them by two examples: the noisy phase dynamics in a Josephson junction and the noisy Kuramoto model. In presented cases the measured speedup can be as high as $675 \times$ compared to a typical CPU, which corresponds to several billion integration steps per second. This means that calculations which took weeks can now be completed in less than one hour. This brings stochastic simulation to a completely new level, opening for research a whole new range of problems which can now be solved interactively.
\end{abstract}

Key words: Josephson junction, Kuramoto, graphics processing unit, advanced computer architecture, numerical integration, diffusion, stochastic differential equation, CUDA, Tesla, NVIDIA

\section{Introduction}

The numerical integration of stochastic differential equations (SDEs) is a valuable tool for analysis of a vast diversity of problems in physics, ranging from equilibrium transport in molecular motors [1, phase dynamics in Josephson junctions [2, 3], stochastic resonance 4 to dissipative particle dynamics [5] to finance [6]. Stochastic simulation, as it is referred to as, is specially interesting when the dimensionality of the problem is larger than three, and in that case it is often the only effective numerical method. A prominent example of this is the stochastic variation of molecular dynamics: Brownian dynamics.

Direct stochastic simulations require a significant computational effort, and therefore merely a decade ago have been used mostly as validation tools. The precise numerical results in theory of low-dimensional stochastic problems were coming from solutions of the corresponding Fokker-Planck equations. Many different sophisticated, but often complicated, tools have been applied: spectral methods [7, 8, 9, finite element methods [10] and numerical path integrals [11, 12. 
Stochastic simulation gained acceptance due to its straightforward implementation and generic robustness with respect to different sorts of problems. The continuous increase of the efficiency of available computer hardware has been acting in favour of stochastic simulation, making it increasingly more popular. The recent evolution of computer architectures towards multiprocessor and multicore platforms also resulted in improved performance of stochastic simulation. Let us note that in the case of a low-dimensional system, stochastic simulation often uses ensemble averaging to obtain the values of observables, which in turn is an example of a so-called ,embarrassingly parallel problem” and it can, though with embarrassment, directly benefit from a parallel architecture. In other cases, mostly where a large number of interacting subsystems are investigated, the implementation of the problem on a parallel architecture is less trivial, but still possible.

The recent emergence of techniques collectively known as general-purpose computing on graphics processing units (GPUs) has caused a breakthrough in computational science. The current state of the art GPUs are now capable of performing computations at a rate of about 1 TFLOPS per single silicon chip. It must be stressed that 1 TFLOPS is a performance level which only in 1996 was achievable exclusively by huge and expensive supercomputers such as the ASCI Red Supercomputer (which had a peak performance of 1.8 TFLOPS [13]) The numerical simulations of SDEs can easily benefit from the parallel GPU architecture. This however requires careful redesign of the employed algorithms and in general cannot be done automatically. In this paper we present a practical introduction to solving SDEs on NVIDIA GPUs using Compute Unified Device Architecture (CUDA) [14 based on two examples: the model of phase diffusion in a Josephson junction and the Kuramoto model of coupled phase oscillators.

The paper is organized as follows: first, we briefly introduce the features and capabilities of the NVIDIA CUDA environment and describe the two physical models, then we present the implementation of stochastic algorithms and compare their efficiency with a corresponding pure-CPU implementation executed on an Intel Core2 Duo E6750 processor. We also provide the source code 15] of three small example programs: PROG1, PROG2, and PROG3, which demonstrate the techniques described in the paper. They can easily be extended to a broad range of problems involving stochastic differential equations.

\section{The CUDA environment}

CUDA (Compute Unified Device Architecture) is the name of a general purpose parallel computing architecture of modern NVIDIA GPUs. The name $C U D A$ is commonly used in a wider context to refer to not only the hardware architecture of the GPU, but also to the software components used to program that hardware. In this sense, the CUDA environment also includes the NVIDIA CUDA compiler and the system drivers and libraries for the graphics adapter.

From the hardware standpoint, CUDA is implemented by organizing the GPU around the concept of a streaming multiprocessor (SM). A modern NVIDIA 


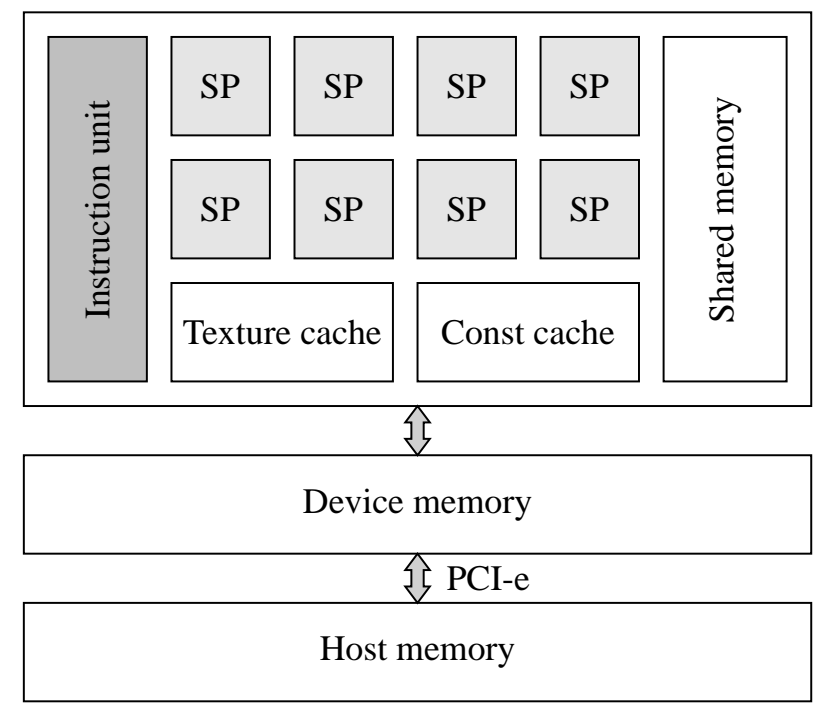

Figure 1: A schematic view of a CUDA streaming multiprocessor with 8 scalar processor cores.

GPU contains tens of multiprocessors. A multiprocessor consists of 8 scalar processors (SPs), each capable of executing an independent thread (see Fig. 1). The multiprocessors have four types of on-chip memory:

- a set of 32-bit registers (local, one set per scalar processor)

- a limited amount of shared memory (16 kB for devices having Compute Capability 1.3 or lower, shared between all SPs in a MP)

- a constant cache (shared between SPs, read-only)

- a texture cache (shared between SPs, read-only)

The amount of on-chip memory is very limited in comparison to the total global memory available on a graphics device (a few kilobytes vs hundreds of megabytes). Its advantage lies in the access time, which is two orders of magnitude lower than the global memory access time.

The CUDA programming model is based upon the concept of a kernel. A kernel is a function that is executed multiple times in parallel, each instance running in a separate thread. The threads are organized into one-, two- or threedimensional blocks, which in turn are organized into one- or two-dimensional grids. The blocks are completely independent of each other and can be executed in any order. Threads within a block however are guaranteed to be run on a single multiprocessor. This makes it possible for them to synchronize and share information efficiently using the on-chip memory of the SM.

In a device having Compute Capability 1.2 or higher, each multiprocessor is capable of concurrently executing 1024 active threads [16]. In practice, the 
number of concurrent threads per SM is also limited by the amount of shared memory and it thus often does not reach the maximum allowed value.

The CUDA environment also includes a software stack. For CUDA v2.1, it consists of a hardware driver, system libraries implementing the CUDA API, a CUDA $\mathrm{C}$ compiler and two higher level mathematical libraries (CUBLAS and CUFFT). CUDA $\mathrm{C}$ is a simple extension of the $\mathrm{C}$ programming language, which includes several new keywords and expressions that make it possible to distinguish between host (i.e. CPU) and GPU functions and data.

\section{Specific models}

In this work, we study the numerical solution of stochastic differential equations modeling the dynamics of Brownian particles. The two models we concentrate upon are of particular interest in many disciplines and illustrate the flexibility of the employed methods of solution.

The first model describes a single Brownian particle moving in a symmetric periodic potential $V(x)=\sin (2 \pi x)$ under the influence of a constant bias force $f$ and a periodic unbiased driving with amplitude $a$ and frequency $\omega$ :

$$
\ddot{x}+\gamma \dot{x}=-V^{\prime}(x)+a \cos (\omega t)+f+\sqrt{2 \gamma k_{B} T} \xi(t)
$$

where $\gamma$ is the friction coefficient and $\xi(t)$ is a zero-mean Gaussian white noise with the auto-correlation function $\langle\xi(t) \xi(s)\rangle=\delta(t-s)$ and noise intensity $k_{B} T$.

Equation 1 is known as the Stewart-McCumber model [3] describing phase differences across a Josephson junction. It can also model a rotating dipole in an external field, a superionic conductor or a charge density wave. It is particularly interesting since it exhibits a wide range of behaviors, including chaotic, periodic and quasi-periodic motion, as well as the recently detected phenomenon of absolute negative mobility [17, 18].

The second model we analyze is that of $N$ globally interacting overdamped Brownian particles, with the dynamics of the $i$-th particle described by:

$$
\begin{array}{r}
\gamma \dot{x_{i}=} \omega_{i}+\sum_{j=1}^{N} K_{i j} \sin \left(x_{j}-x_{i}\right)+ \\
\sqrt{2 \gamma k_{B} T} \xi_{i}(t), i=1, \ldots, N
\end{array}
$$

This model is known as the Kuramoto model [19] and is used as a simple paradigm for synchronization phenomena. It has found applications in many areas of science, including neural networks, Josephson junction and laser arrays, charge density waves and chemical oscillators.

\section{Numerical solution of SDEs}

Most stochastic differential equations of practical interest cannot be solved analytically, and thus direct numerical methods have to be used to obtain the 
solutions. Similarly as in the case of ordinary differential equations, there is an abundance of methods and algorithms for solving stochastic differential equations. Their detailed description can be found in references: [20, 21, 22, 23, 24, 25.

Here, we present the implementation of a standard stochastic algorithm on the CUDA architecture in three distinctive cases:

1. Multiple realizations of a system are simulated, and an ensemble average is performed to calculate quantities of interest. The large degree of parallelism inherent in the problem makes it possible to fully exploit the computational power of CUDA devices with tens of multiprocessors capable of executing hundreds of threads simultaneously. The example system models the stochastic phase dynamics in a Josephson junction and is implemented in program PROG1 (the source code is available in [15]).

2. The system consists of $N$ globally interacting particles. In each time step $N^{2}$ interaction terms are calculated. The example algorithm is named PROG2 and solves the Kuramoto model (Eq. 2.)

3. The system consists of $N$ globally interacting particles as in the previous case but the interaction can be expressed in terms of a parallel reduction operation, which is much more efficient than PROG2. The example algorithm in PROG3 also solves the Kuramoto model (Eq. 2.)

We will now outline the general patterns used in the solutions of all models. We start with the model of a single Brownian particle, which will form a basis upon which the solution of the more general model of $N$ globally interacting particles will be based.

\subsection{Ensemble of non-interacting stochastic systems}

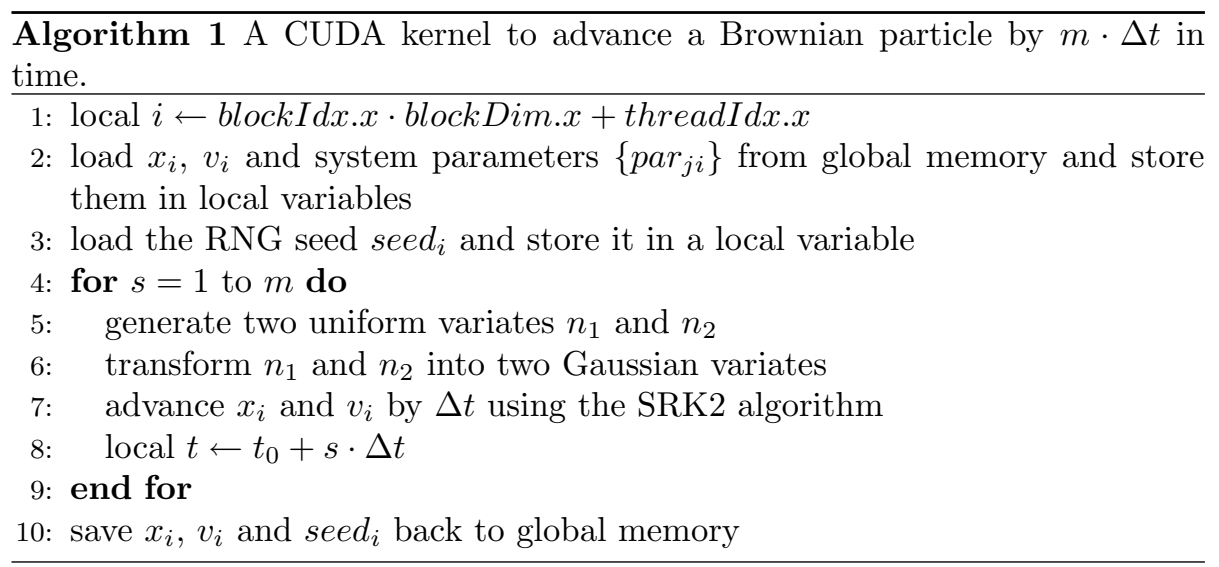

For the Josephson junction model described by Eq. 1 we use a single CUDA kernel, which is responsible for advancing the system by a predefined number of timesteps of size $\Delta t$. 


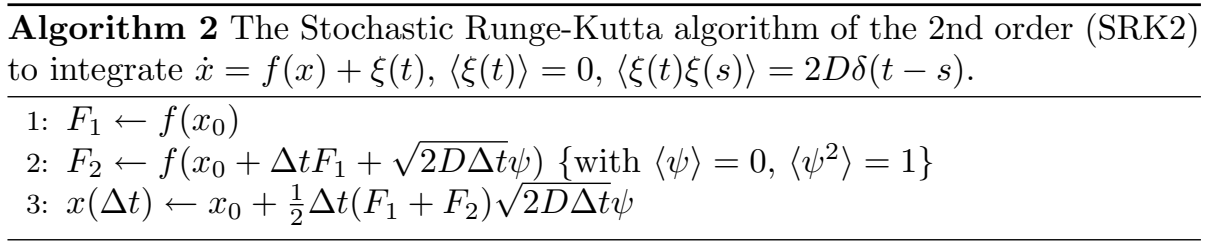

We employ fine-grained parallelism - each path is calculated in a separate thread. For CUDA devices, it makes sense to keep the number of threads as large as possible. This enables the CUDA scheduler to better utilize the available computational power by executing threads when other ones are waiting for global memory transfers to be completed [16. It also ensures that the code will execute efficiently on new GPUs, which, by the Moore's law, are expected to be capable of simultaneously executing exponentially larger numbers of threads. We have found that calculating $10^{5}$ independent realizations is enough to obtain a satisfactory level of convergence and that further increases of the number of paths do not yield better results (see Fig. 5).

In order to increase the number of threads, we structured our code so that Eq. 1 is solved for multiple values of the system parameters in a single run. The default setup calculates trajectories for 100 values of the amplitude parameter a. This makes it possible to use our code to efficiently analyze the behavior of the system for whole regions of the parameter space $\{a, \omega, \gamma\}$.

Multiple timesteps are calculated in a single kernel invocation to increase the efficiency of the code. We observe that usually only samples taken every $M$ steps are interesting to the researcher running the simulation, the sampling frequency $M$ being chosen so that the relevant information about the analyzed system is retained. In all following examples $M=100$ is used. It should be noted that the results of the intermediate steps do not need to be copied to the host (CPU) memory. This makes it possible to limit the number of global memory accesses in the CUDA threads. When the kernel is launched, path parameters $x, v=\dot{x}$ and $a$ are loaded from the global memory and are cached in local variables. All calculations are then performed using these variables and at the end of the kernel execution, their values are written back to the global memory.

Each path is associated with its own state of the random number generator (RNG), which guarantees independence of the noise terms between different threads. The initial RNG seeds for each thread are chosen randomly using a standard integer random generator available on the host system. Since CUDA does not provide any random number generation routines by default, we implemented a simple xor-shift RNG as a CUDA device function. In our kernel, two uniform variates are generated per time step and then transformed into Gaussian variates using the Box-Muller transform. The integration is performed using a Stochastic Runge-Kutta scheme of the 2nd order, which uses both Gaussian variates for a single time step.

In the example in Fig. 2 we present the results coming from the simultaneous 


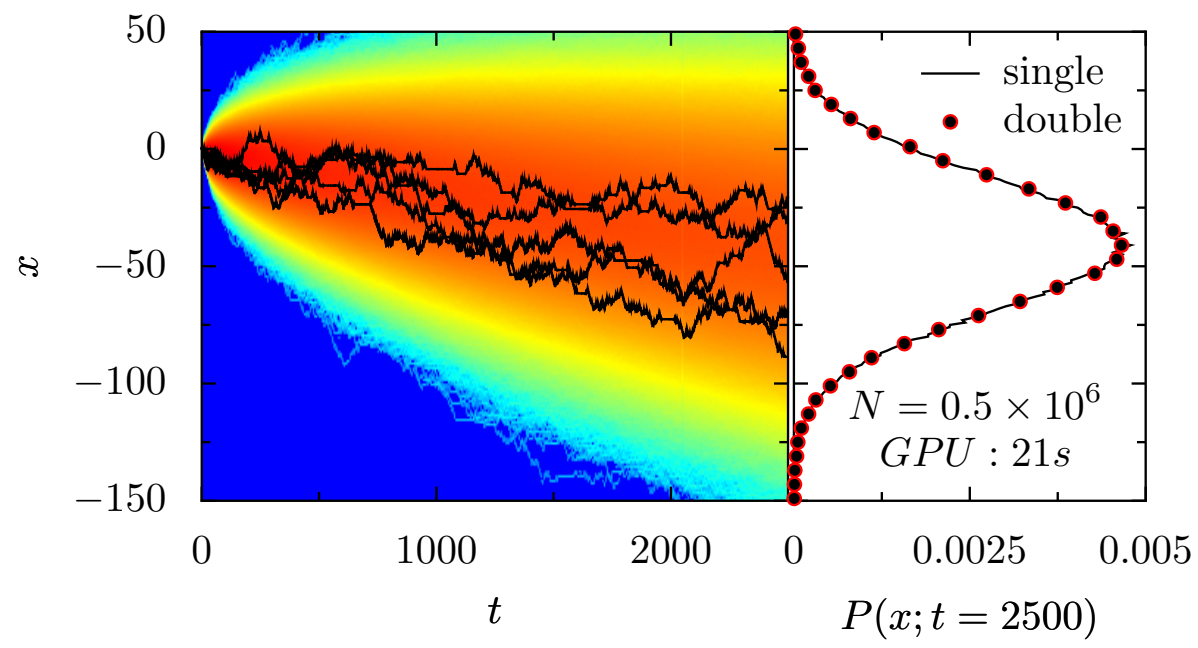

Figure 2: The ensemble of 524288 Brownian particles, modeling the noisy dynamics of phase in a Josephson junction described by Eq. 1 is simulated for time $t \in\left(0,2000 \frac{2 \pi}{\omega}\right)$ with time step $\Delta t=0.01 \frac{2 \pi}{\omega}$. On the left panel sample trajectories are drawn with black lines and the background colors represent the coarse-grained (averaged over a potential period) density of particles in the whole ensemble. The right panel shows the coarse-grained probability distribution of finding a particle at time $t=2000 \frac{2 \pi}{\omega}$ obtained by means of a histogram with 200 bins. The histogram is calculated with both single and double precision on a GPU with Compute Capability v1.3. The same calculation has also been performed on the CPU but their identical results are not presented for clarity purposes. The total simulation times were: 20 seconds and 13 minutes on NVIDIA Tesla 1060C when using single and double precision floating-point arithmetics, respectively. The CPU-based version of the same algorithm needed over three hours. Used parameters: $a=4.2, \gamma=0.9, \omega=4.9, D_{0}=0.001, f=0.1$ correspond to the anomalous response regime (cf. 17]). 
solution of $N=2^{19}=524288$ independent Eqs. 1 for the same set of parameters. The total simulation time was less than 20 seconds. In this case the CUDA platform turns out to be extremely effective, outperforming the CPU by a factor of 675 . In order to highlight the amount of computation, let us note that the size of the intermediate file with all particle positions used for generation of the background plot was about $30 \mathrm{~GB}$.

\section{2. $N$ globally interacting stochastic systems}

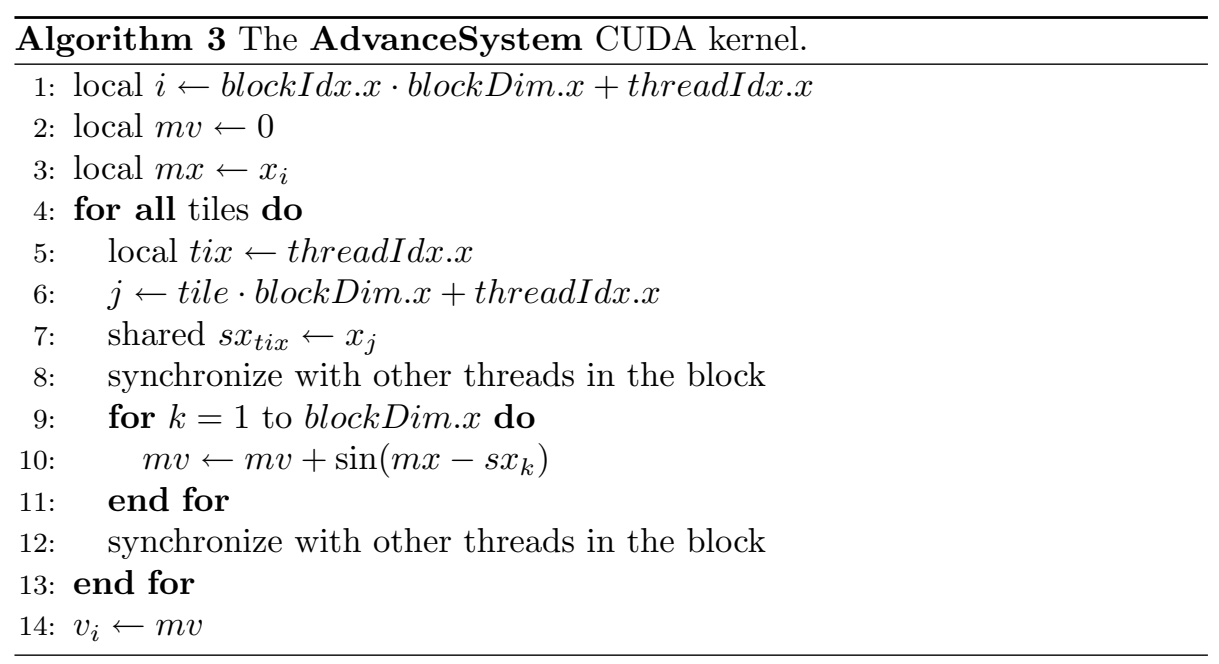

For the general Kuramoto model described by Eqs. 2 or other stochastic systems of $N$ interacting particles, the calculation of $\mathcal{O}\left(N^{2}\right)$ interaction terms for all pairs $\left(x_{j}, x_{i}\right)$ is necessary in each integration step. In this case the program PROG2 is split into two parts, implemented as two CUDA kernels launched sequentially. The first kernel, called UpdateRHS calculates the right hand side of Eq. 2 for every $i$. The second kernel AdvanceSystem actually advances the system by a single step $\Delta t$ and updates the positions of all particles. In our implementation the second kernel uses a simple first-order Euler scheme. It is straightforward to modify the program to implement higher-order schemes by interleaving calls to the UpdateRHS kernel with calls to kernels implementing the sub-steps of the scheme.

The UpdateRHS kernel is organized around the concept of tiles, introduced in [26]. A tile is a group of $T$ particles interacting with another group of $T$ particles. Threads are executed in blocks of size $T$ and each block is always processing a single tile. There is a total of $N / T$ blocks in the grid. The $i$-th thread computes the interaction of the $i$-th particle with all other particles.

The execution proceeds as follows. The $i$-th thread loads the position of the $i$-th particle and caches it as a local variable. It then loads the position of another particle from the current tile, stores it in shared memory and synchronizes with other threads in the block. When this part is completed, the 


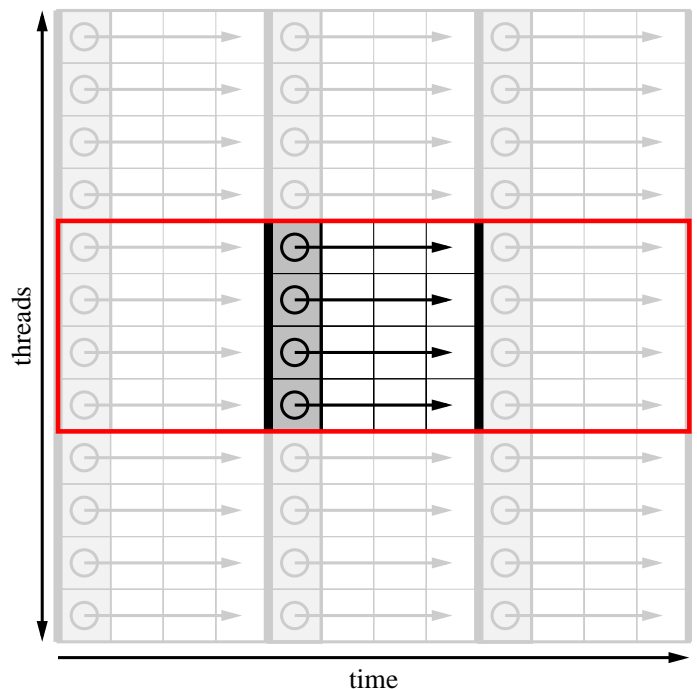

Figure 3: All-pairs interaction of 12 particles calculated using the tile-based approach with 9 tiles of size $4 \times 4$. The chosen number of particles and the size of the tiles are made artificially low for illustration purposes only. A small square represents the computation of a single particle-particle interaction term. The highlighted part of the schematic depicts a single tile. The bold lines represent synchronization points where data is loaded into the shared memory of the block. The filled squares with circles represent the start of computation for a new tile. Threads in the red box are executed within a single block. 


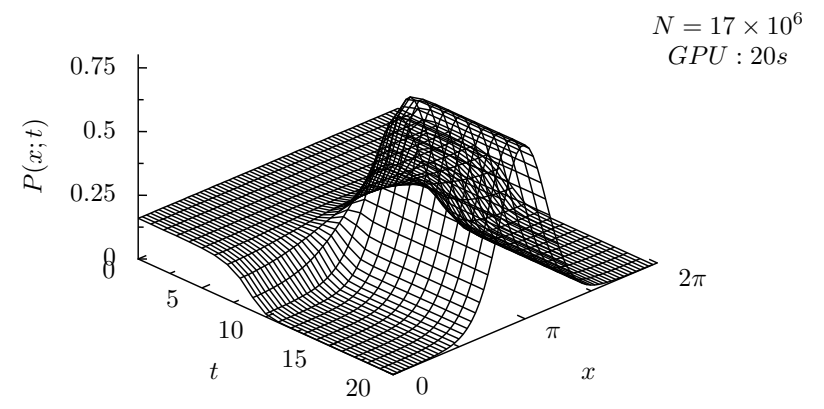

Figure 4: An example result of the integration of the Kuramoto system (Eq. 2). The time evolution of the probability density $P(x ; t)$ is shown for $\omega_{i}=0, K_{i j}=4, T=1$. The density is a position histogram of $2^{24}$ particles. The total time of simulation was approximately 20 seconds using the single precision capabilities of NVIDIA Tesla C1060.

positions of all particles from the current tile are cached in the shared memory. The computation of the interaction is then commenced, with the $i$-th thread computing the interaction of the $i$-th particle with all particles from the current tile. Afterwards, the kernel advances to the following tile, the positions stored in shared memory are replaced with new ones, and the whole process repeats.

This approach might seem wasteful since it computes exactly $N^{2}$ interaction terms, while only $(N-1) N / 2$ are really necessary for a symmetric interaction. It is however very efficient, as it minimizes global memory transfers at the cost of an increased number of interaction term computations. This turns out to be a good trade-off in the CUDA environment, as global memory accesses are by far the most costly operations, taking several hundred clock cycles to complete. Numerical computations are comparatively cheap, usually amounting to just a few clock cycles.

The special form of the interaction term in the Kuramoto model when $K_{i j}=K=$ const, allows us to significantly simplify the calculations. Using the identity:

$$
\begin{array}{r}
\sum_{j=1}^{N} \sin \left(x_{j}-x_{i}\right)= \\
\cos \left(x_{i}\right) \sum_{j=1}^{N} \sin \left(x_{j}\right)-\sin \left(x_{i}\right) \sum_{j=1}^{N} \cos \left(x_{j}\right)
\end{array}
$$

we can compute two sums: $\sum_{j=1}^{N} \sin \left(x_{j}\right)$ and $\sum_{j=1}^{N} \cos \left(x_{j}\right)$ only once per integration step, which has a computational cost of $\mathcal{O}(N)$. The calculation of the sum of a vector of elements is an example of the vector reduction operation, which can be performed very efficiently on the CUDA architecture. Various methods of implementation of such an operation are presented in the sample code included in the CUDA SDK 2.1 [27]. The integration of the Kuramoto system taking advantage of Eq. 3 and using a simple form of a parallel reduction is implemented in PROG3. 
In Fig. 4 we present a solution of the classical Kuramoto system described by Eqs. 2 for parameters as in Fig. 10 of the review paper [19. In this case we apply the program PROG3 which makes use of the relation from Eq. 3 . The number of particles $N=2^{24} \approx 16.8 \cdot 10^{6}$ and the short simulation time clearly demonstrate the power of the GPU for this kind of problems.

\section{Note on single precision arithmetics}

The fact that the current generation of CUDA devices only implements single precision operations in an efficient way is often considered a significant limitation for numerical calculations. We have found out that for the considered models this does not pose a problem. Figure 2 presents sample paths and position distribution functions of a Brownian particle whose dynamics is determined by Eq. 1] (colored background on the left panel and right panel). Let us note that we present coarse-grained distribution functions where the position is averaged over a potential period by taking a histogram with bin size being exactly equal to the potential period. We observe that the use of single precision floating-point numbers does not significantly impact the obtained results. Results obtained by

single precision calculations even after a relatively long time $t=2000 \frac{2 \pi}{\omega}$ differ from their double precision counterparts only up to the statistical error, which in this case can be estimated by the fluctuations of the relative particle number in a single histogram bin. Since in the right panel of Fig. 2 we have approximately $10^{4}$ particles in one bin, the error is of the order of $1 \%$. If time-averaged quantities such as the asymptotic velocity $\langle\langle v\rangle\rangle=\lim _{t \rightarrow \infty}\langle v(t)\rangle$ are calculated, the differences are even less pronounced. However, the single and double precision programs produce different individual trajectories as a direct consequence of the chaotic nature of the system given by Eq. 1. Moreover, we have noticed that even when changing between GPU and CPU versions of the same program, the individual trajectories diverged after some time. The difference between paths calculated on the CPU and the GPU, using the same precision level, can be explained by differences in the floating-point implementation, both in the hardware and in the compilers.

When doing single precision calculations special care must be taken to ensure that numerical errors are not needlessly introduced into the calculations. If one is used to having all variables defined as double precision floating-point numbers, as is very often the case on a CPU, it is easy to forget that operations which work just fine on double precision numbers might fail when single precision numbers are used instead. For instance, consider the case of keeping track of time in a simulation by naively increasing the value of a variable $t$ by a constant $\Delta t$ after every step. By doing so, one is bound to hit a problem when $t$ becomes large enough, in which case $t$ will not change its value after the addition of a small value $\Delta t$, and the simulation will be stuck at a single point in time. With double precision numbers this issue becomes evident when there is a difference of 17 orders of magnitude between $t$ and $\Delta t$. With single precision numbers, a 8-orders-of-magnitude difference is enough to trigger the problem. It means that if, for instance, $t$ is $10^{5}$ and $\Delta t$ is $10^{-4}$, the addition will no longer work 

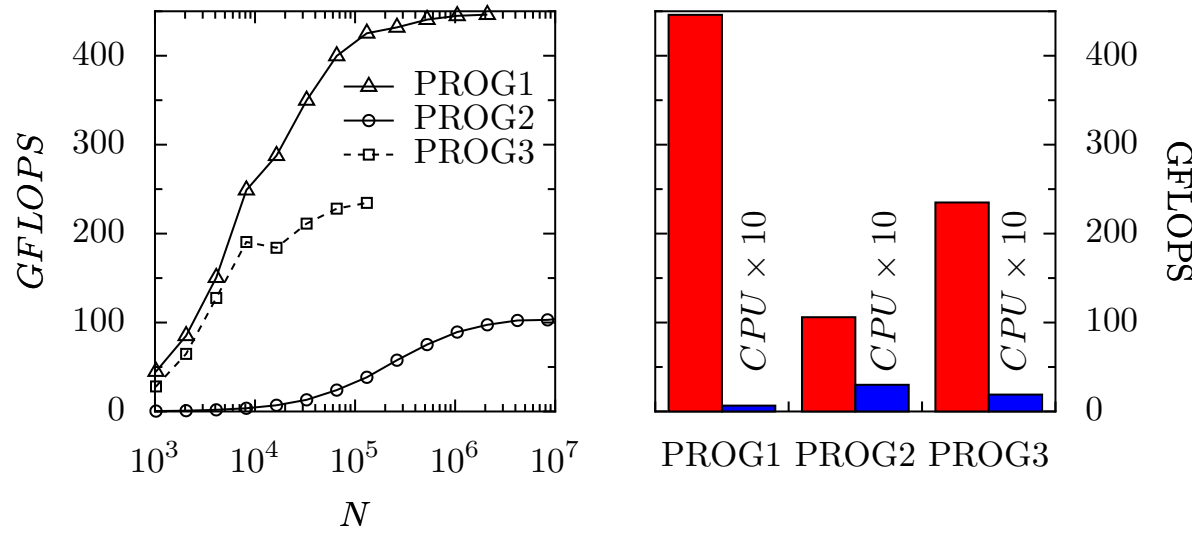

Figure 5: (Left panel) Performance estimate for the programs PROG1-PROG3 as a function of the number of particles $N$. (Right panel) Performance estimate for the programs PROG1PROG3 on an Intel Core2 Duo E6750 CPU and NVIDIA Tesla C1060 GPU. We have counted $79,44+6 N$ and 66 operations per one integration step of programs PROG1, PROG2 and PROG3, respectively.

as expected. $10^{5}$ and $10^{-4}$ are values not uncommon in simulations of the type we describe here, hence the need for extra care and reformulation of some of the calculations so that very large and very small quantities are not used at the same time. In our implementations, we avoided the problem of spurious addition invariants by keeping track of simulation time modulo the system period $2 \pi / \omega$. This way, the difference between $t$ and $\Delta t$ was never large enough to cause any issues.

\section{Performance evaluation}

In order to evaluate the performance of our numerical solution of Eqs. 1 and 2 we first implemented Algs. 3 and 1 using the CUDA Toolkit v2.1. We then translated the CUDA code into $\mathrm{C}++$ code by replacing all kernel invocations with loops and removing unnecessary elements (such as references to shared memory, which does not exist on a CPU).

We used the NVIDIA CUDA Compiler (NVCC) and GCC 4.3.2 to compile the CUDA code and the Intel C++ Compiler (ICC) v11.0 for Linux to compile the $\mathrm{C}++$ version. We have determined through numerical experiments that enabling floating-point optimizations significantly improves the performance of our programs (by a factor of 7 on CUDA) and does not affect the results in a quantitative or qualitative way. We have therefore used the $-f$ ast $-f p$-model fast=2 ICC options and --use_fast_math in the case of NVCC.

All tests were conducted on recent GNU/Linux systems using the following hardware:

- for the CPU version: Intel Core2 Duo E6750 @ 2.66GHz and 2 GB RAM (only a single core was used for the calculations) 
- for the GPU version: NVIDIA Tesla C1060 installed in a system with Intel Core2 Duo CPU E2160 @ 1.80GHz and 2 GB RAM

Our tests indicate that speedups of the order of 600 and 100 are possible for the models described by Eqs. 1 and 2, respectively. The performance gain is dependent on the number of paths used in the simulation. Figure 5 shows that it increases monotonically with the number of paths, and then saturates at a number dependent on the used model: 450 and 106 GFLOPS for the Eqs. 1 and 2, respectively (which corresponds to speedups: 675 and 106). The saturation point indicates that for the corresponding number of particles the full computational resources of the GPU are being exploited.

The problem of lower performance gain for small numbers of particles could be rectified by dividing the computational work between threads in a different way, i.e. by decreasing the amount of calculations done in a single thread, while increasing the total number of threads. This is a relatively straightforward thing to do, but it increases the complexity of the code. We decided not to do it since for models like 1 and 2 one is usually interested in calculating observables for whole ranges of system parameters. Instead of modifying the code to run faster for lower number of paths, one can keep the number of paths low but run the simulation for multiple system parameters simultaneously, which results in a higher number of threads.

\section{Conclusions}

In this paper we have demonstrated the suitability of a parallel CUDA-based hardware platform for solving stochastic differential equations. The observed speedups, compared to CPU versions, reached an astonishing value 670 for noninteracting particles and 120 for a globally coupled system. We have also shown that for this kind of calculations single precision arithmetics poses no problems with respect to accuracy of the results, provided that some kind of operations, such as adding small and large numbers, are avoided.

The availability of cheap computer hardware which is over two orders of magnitude faster clearly announces a new chapter in high performance computing. Let us note that the development of stream processing technology for general-purpose computing has just started and its potential is surely not yet fully revealed. In order to take advantage of the new hardware architecture, the software and its algorithms must be substantially redesigned.

\section{Appendix: Estimation of FLOPS}

We counted the floating-point operations performed by the kernels in our code, and the results in the form of the collective numbers of elementary operations are presented in Table 1. The number of MAD (Multiply and Add) operations can vary, depending on how the compiler processes the source code. 
Table 1: Number of elementary floating-point operations performed per one time step in the AdvanceSystem kernel for Eq. 1]

\begin{tabular}{|c|c|c|c|}
\hline count & type & FLOPs & totalFLOPs \\
\hline \hline 22 & multiply, add & 1 & 22 \\
\hline 11 & MAD & 1 & 11 \\
\hline 2 & division & 4 & 8 \\
\hline 3 & sqrt & 4 & 12 \\
\hline 1 & sin & 4 & 4 \\
\hline 5 & $\cos$ & 4 & 20 \\
\hline 1 & $\log$ & 2 & 2 \\
\hline \multicolumn{5}{|c|}{ TOTAL: } & $\mathbf{7 9}$ \\
\hline
\end{tabular}

For the purposes of our performance estimation, we assumed the most optimistic version. A more conservative approach would result in a lower number of MADs, and correspondingly a higher total number of GFLOPS.

The amount of FLOPs for functions such as sin, log, etc. is based on [16, assuming 1 FLOP for elementary arithmetical operations like addition and multiplication and scaling the FLOP estimate for complex functions proportionately to the number of processor cycles cited in the manual. The numbers of floatingpoint operations are summarized in Table 1.

On a Tesla C1060 device our code PROG1 evaluates $6.178 \cdot 10^{9}$ time steps per second. The cost of each time step is 79 FLOPs, which implies that the overall performance estimate accounts for 490 GFLOPS.

In the case of PROG2 the number of operations per one integration step depends on the number of particles $N$. A similar operation count as the one presented in Table 1 resulted in the formula $44+6 N$ FLOPs per integration step.

\section{References}

[1] Reimann, P., Physics Reports 361 (2002) 57.

[2] Kostur, M., Machura, L., Talkner, P., Hänggi, P., and Łuczka, J., Physical Review B (Condensed Matter and Materials Physics) 77 (2008) 104509.

[3] Kautz, R. L., Reports on Progress in Physics 59 (1996) 935.

[4] Gammaitoni, L., Hänggi, P., Jung, P., and Marchesoni, F., Rev. Mod. Phys. 70 (1998) 223.

[5] Groot, R. and Warren, P., J. Chem. Phys. 107 (1997) 4423.

[6] McLeish, D. L., Monte Carlo Simulation and Finance, John Wiley and Sons, 2005.

[7] Bartussek, R., Reimann, P., and Hänggi, P., Phys. Rev. Lett. 76 (1996) 1166. 
[8] Lindner, B., Schimansky-Geier, L., Reimann, P., Hänggi, P., and Nagaoka, M., Phys. Rev. E 59 (1999) 1417.

[9] Kalmykov, Y. P., Phys. Rev. E 61 (2000) 6320.

[10] Kostur, M., Internat. J. Modern Phys. C 13 (2002) 1157.

[11] Yu, J. and Lin, Y., Internat. J. Non-Linear Mech. 39 (2004) 1493.

[12] Naess, A., Dimentberg, M. F., and Gaidai, O., Physical Review E (Statistical, Nonlinear, and Soft Matter Physics) 78 (2008) 021126.

[13] http://www.computermuseum.li/testpage/asci-red-supercomputer.htm.

[14] Nvidia cuda webpage, http://www.nvidia.com/object/cuda_home.html.

[15] Source code of all examples can be found on http://fizyka.us.edu.pl/cuda.

[16] Corporation, N., Nvidia cuda programming guide v2.1, available from nvidia cuda webpage, http://www.nvidia.com/object/cuda_home.html, 2008.

[17] Machura, L., Kostur, M., Talkner, P., Łuczka, J., and Hänggi, P., Physical Review Letters 98 (2007) 040601.

[18] Speer, D., Eichhorn, R., and Reimann, P., EPL (Europhysics Letters) 79 (2007) 10005 (5pp).

[19] Acebron, J. A., Bonilla, L. L., Vicente, C. J. P., Ritort, F., and Spigler, R., Reviews of Modern Physics 77 (2005) 137.

[20] Mannella, R. and Palleschi, V., Phys. Rev. A 40 (1989) 3381.

[21] Mannella, R., Internat. J. Modern Phys. C 13 (2002) 1177.

[22] Sancho, J. M., Miguel, M. S., Katz, S. L., and Gunton, J. D., Phys. Rev. A 26 (1982) 1589.

[23] Fox, R. F., Gatland, I. R., Roy, R., and Vemuri, G., Phys. Rev. A 38 (1988) 5938.

[24] Honeycutt, R. L., Phys. Rev. A 45 (1992) 600.

[25] Kloeden, P. E. and Platen, E., Numerical Solution of Stochastic Differential Equations (Stochastic Modelling and Applied Probability), Springer, 2000.

[26] L. Nyland, M. Harris, J. P., GPU Gems 3 - Fast N-body simulation with CUDA, chapter 31, pages 677-695, Addison-Wesley Professional, 2007.

[27] Nvidia cuda software development kit, available from nvidia cuda webpage, http://www.nvidia.com/object/cuda_home.html. 